\title{
Minimal predicted distance: a kinematic cue to investigate collision avoidance between walkers
}

\author{
A.-H. Olivier ${ }^{1}$, A. Marin ${ }^{2}$, A. Crétual ${ }^{2}$, J. Pettré$^{1}$ \\ Equipe MIMETIC : ${ }^{I}$ INRIA Rennes-Bretagne Atlantique, France $;{ }^{2} M 2 S$, UEB, University of Rennes 2, France
}

Keywords: Interaction; collision avoidance; locomotion; distance; anticipatory locomotor control.

\section{Introduction}

Collision-free navigation requires avoiding static and moving obstacles as well as other humans. Previous studies focused on the avoidance of static $[1,2]$ or passive moving obstacles such as a mannequin [3,4]. Collision avoidance between human walkers was only studied in a frontal task to investigate the influence of gender and height on the lateral deviation and clearance distance [5].

Our objective was to identify the conditions that lead to avoidance manoeuvres in locomotor trajectories of two walkers. Based on the assumption of a reciprocal interaction, we suggested a mutual variable, the Minimum Predicted Distance (MPD), which emphasises the risk of collision and describes the general collision avoidance behaviour.

\section{Methods}

30 participants volunteered for this experiment $\left(11\right.$ \% $\left.19{ }^{\lambda}\right)$. For each of the 420 trials recorded, 2 participants stood at the corners of the $15 \mathrm{~m}$ side length experimental area (Figure 1A). Their task was to walk to the opposite corner. By synchronizing their start signals, we provoked situations of potential collisions on orthogonal trajectories. The variability in natural speeds and reaction times actually changed the exact conditions of the kinematics of interactions, thereby allowing us to study their influence. Occluding walls $(2 \mathrm{~m}$ high by $3 \mathrm{~m}$ long) between corners prevented participants from seeing each other before reaching their natural speeds. The time when participants can see each other was denoted 'tsee'. The time when the distance between participants was minimal ('dmin') was denoted 'tcross'. 3D kinematics were recorded using 12 Vicon MX-40 cameras at a sampling rate of $120 \mathrm{~Hz}$.

We approximated participant's motion as the one of their mid-shoulders and applied a Butterworth lowpass filter $(0.5 \mathrm{~Hz}$ cut-off frequency) to average out the higher frequency stepping oscillations.

Collision avoidance interaction can obviously only occur between tsee and tcross so we temporally normalized all the data between these two instants. At each instant $t$, if no motion adaption was performed by walkers, we can predict their future trajectories as linear extrapolations of their current states:

$$
P_{\text {pred }, 1}(t, u)=P_{1}(t)+(u-t) V_{1}(t)
$$

where $\mathrm{u}$ is a time parameter, $\mathrm{P}_{1}(\mathrm{t})$ the current position and $\mathrm{V}_{1}(\mathrm{t})$ the current velocity vector of participant \#1.
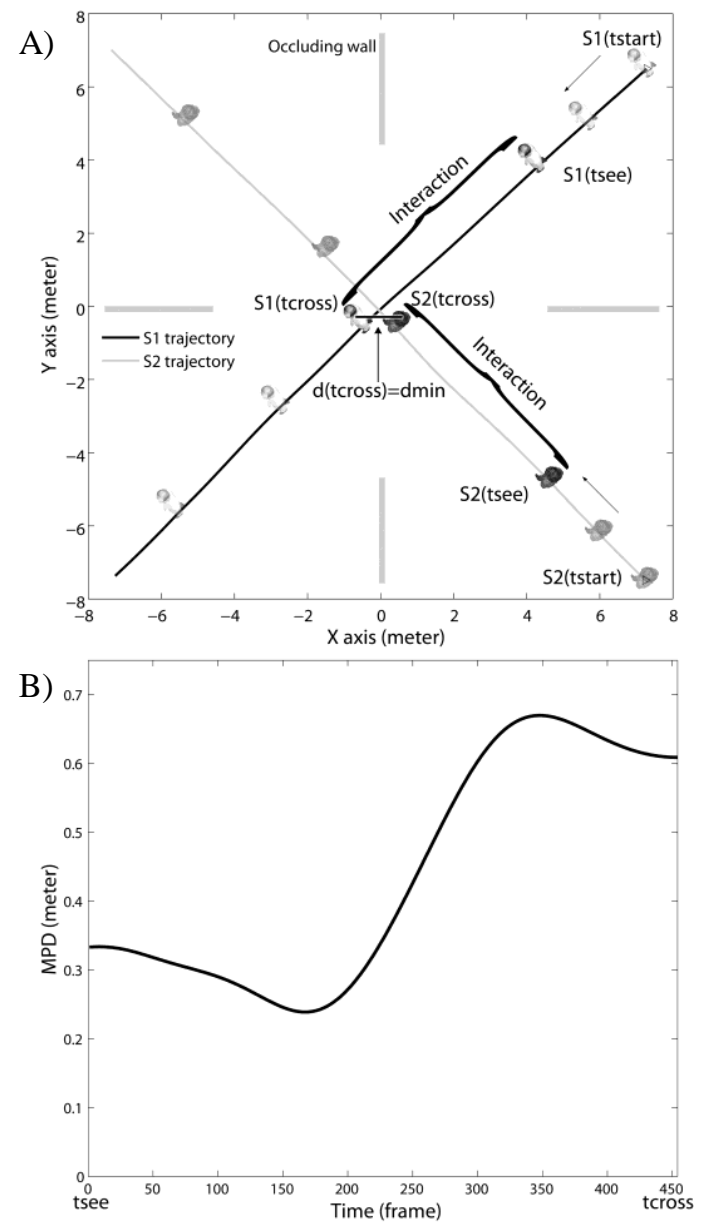

Figure 1: A) Experimental set-up. B) Minimum Predicted Distance over the interaction phase.

We then introduced the Minimal Predicted Distance (MPD). At each instant $t, \operatorname{MPD}(\mathrm{t})$ represented the distance at which participants would meet if they did not perform motion adaptation after this instant:

$$
M P D(t)=\underset{u}{\arg \min }\left\|P_{p r e d, 2}(t, u)-P_{\text {pred }, 1}(t, u)\right\|
$$

As shown in Figure 1B, we studied how $\operatorname{MPD}(\mathrm{t})$ changes over the whole period of interaction [tsee, tcross].

Data were presented with mean \pm SD. All effects were reported at $\mathrm{p}<0.05$. Wilcoxon signed-rank 
tests were used to determine differences between values of MPD at several instants of the interaction.

\section{Results and Discussion}

Minimum distance 'dmin' was $1.09 \mathrm{~m}( \pm 0.47)$ and ranged from 0.41 to $3.48 \mathrm{~m}$. MPD(tsee) ranged from 0 to $3.81 \mathrm{~m}$. We subdivided the dataset in 10 groups of 42 trials according to ascending $\operatorname{MPD}$ (tsee) values. For each group, we computed $\overline{M P D}(t)$ (Fig.2A). When MPD(tsee) was lower than $1 \mathrm{~m}$ (groups 1 to 6), the set of MPD(tcross) values for each group was significantly higher than MPD(tsee) $\left(\mathrm{T}_{1}=0, \mathrm{~T}_{2}=0, \mathrm{~T}_{3}=0, \mathrm{~T}_{4}=0, \mathrm{~T}_{5}=72, \mathrm{~T}_{6}=125 ; \mathrm{df}=41\right.$, $\mathrm{p}<0.01)$. When MPD(tsee) ranged from 1 to $1.5 \mathrm{~m}$, there was no significant difference between the sets of MPD(tcross) and MPD(tsee) $(\mathrm{p}>0.05)$. When MPD(tsee) was higher than $1.5 \mathrm{~m}, \mathrm{MPD}$ (tcross) was significantly smaller than MPD(tsee) $\left(\mathrm{T}_{9}=208\right.$, $\left.\mathrm{T}_{10}=56 ; \mathrm{df}=41, \mathrm{p}<0.05\right)$. These first results show that walkers adapted their trajectories to increase $\operatorname{MPD}(\mathrm{t})$ when $\operatorname{MPD}(\mathrm{tsee})$ was lower than $1 \mathrm{~m}$, i.e., when future collision is predicted by MPD(tsee). These results corroborate previous observations on the preservation of the personal space during interactions [4], but also reveal human ability to accurately predict future crossing distance.
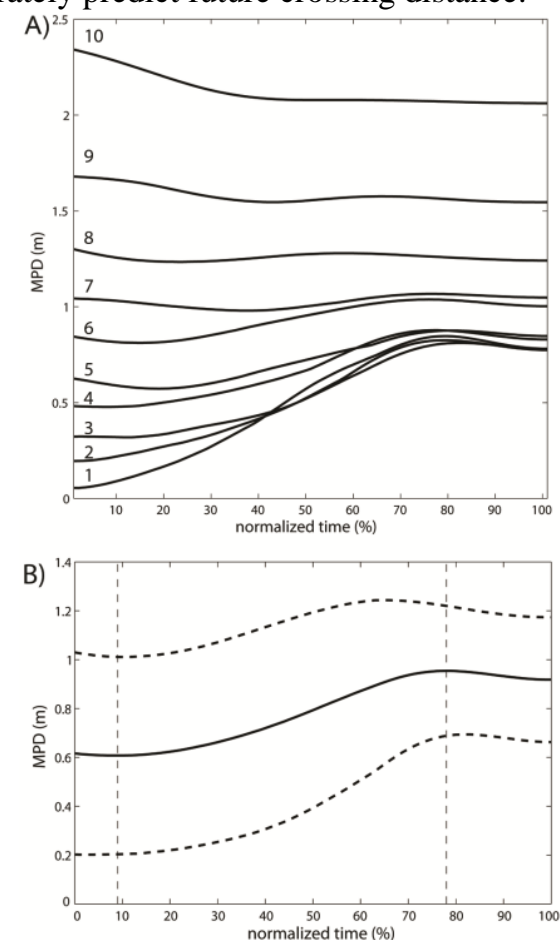

Figure 2: A) $\overline{M P D}(t)$ for 10 groups of ascending

MPD(tsee) values. B) $\overline{M P D}(t)( \pm \mathrm{SD})$ for all trials where $\mathrm{MPD}$ (tsee) $<1 \mathrm{~m}$.

For all trials with MPD(tsee) $<1 \mathrm{~m}$, we computed the overall mean $\overline{M P D}(t)$ (Fig.2B). We then observed three successive phases in time with respect to the sign of its time derivative $\overline{M P D^{\prime}}(t)$. First, the observation phase was between t0\% to $\mathrm{t} 7 \%$ :
$\overline{M P D}(t)$ was constant $(\mathrm{p}>0.05)$. Second, the reaction phase was from $\mathrm{t} 7 \%$ to $\mathrm{t} 79 \%$ : $\overline{M P D^{\prime}}(t)$ was positive and $\overline{M P D}(t)$ significantly increased up to $0.88 \mathrm{~m} \pm 0.22(\mathrm{~T}=258, \mathrm{df}=263, \mathrm{p}<0.01)$. Third, the regulation phase was from $\mathrm{t} 79 \%$ to $\mathrm{t} 100 \%$ : $\overline{M P D^{\prime}}(t)$ was negative and $\overline{M P D}(t)$ slightly decreased to dmin $=0.84 \mathrm{~m} \pm 0.19$, ranging from 0.41 to $1.48 \mathrm{~m}(\mathrm{~T}=-4648, \mathrm{df}=263, \mathrm{p}<0.01)$. This second set of results shows that $\operatorname{MPD}(\mathrm{t})$ captures the temporal structure of interaction (observation, reaction and regulation) and that collision avoidance is anticipated. Indeed, the reaction phase ended $0.8 \mathrm{~s}$ before crossing which indicates that collision avoidance is solved in advance. $\operatorname{MPD}(\mathrm{t})$ is then maintained at constant value during the regulation phase. The duration of this phase $(0.8 \mathrm{~s})$ is close to the duration of a stride. This can be related to the one-stride interval necessary to allow a walker to successfully implement adaptive strategies [6].

\section{Conclusion}

We concluded that walkers are able to accurately predict crossing distances and to react accordingly. We also concluded that avoidance is performed with anticipation, i.e., maneuvers are ended time before interaction ended. However, our analysis focused on the mutual aspects of interaction. Future work is still required to investigate the nature of each walker reactions to avoid collisions.

\section{References}

[1]Jansen SE, Toet A, Werkhoven PJ (2011) Human locomotion through a multiple obstacle environment: strategy changes as a result of visual field limitation. Exp Br Res, 212, 449-456.

[2]Vallis LA, McFadyen BJ (2003) Locomotor adjustments for circumvention of an obstacle in the travel path. Exp Brain Res, 152, 409-414.

[3]Cinelli ME, Patla AE (2007) Travel path conditions dictate the manner in which individuals avoid collisions. Gait Posture, 26, 186-193.

[4]Gérin-Lajoie M, Richards C, McFadyen B (2005) The negociation of stationary and moving obstructions during walking: anticipatory locomotor adaptations and preservation of personal space. Motor Control, 9, 242-269.

[5]van Basten BJ, Jansen SE, Karamouzas I (2009) Exploiting motion capture to enhance avoidance behaviour in games. Lect Notes Comput Sci, 5884, 29-40.

[6]Patla AE (1997) Understanding the role of vision in the control of human locomotion. Gait Posture, 5, 54-69.

\section{Acknowledgments}

This study was funded by the French ANR PSIROB Locanthrope project and the European FP7-ICT2009C Tango Project ( $\left.n^{\circ} 249858\right)$. 